\title{
Docking control of the flexible riser end in an offshore loading system
}

\author{
STEINAR SAELID +
}

Keywords: Kalman filtering, marine operations, hydrodynamics.

The SUBLOAD system for offshore loading of oil directly from a seafloor terminal by a flexible riser deployed from a dynamically positioned shuttle tanker is briefly described.

The flexible riser is terminated at the lower end by a Lower Riser Package (LRP). The LRP is equipped with thrusters to control the lower riser end position during the docking- and LRP connection-phase.

A mathematical model of the vessel/riser/LRP system is given, and a simulation model is used for design of some of the LRP parameters (weight, buoyancy, metacentre). This is done in order to minimize the thrust force requirement for docking control purposes.

Finally a docking control system is designed based on a simplified model of the LRP dynamics. The control system uses feedback from LRP-states as well as feedforward from estimated force components acting directly on the LRP. The system is adaptive in the sense that the dominating wave force frequency is continuously estimated and used by the control algorithm.

The control system is tested against a realistic vessel/riser/LRP simulator, and the control performance is shown to be superior compared to a more direct PID-based control system.

\section{Introduction}

The sub-load system proposed is an offshore loading system based on dynamically positioned shuttle tankers loading oil directly from a seaffoor terminal through a flexible continuous riser.

The seaffoor terminal is mounted on a gravity base at the end of an export pipeline from a production/process platform at least 2000 metres away. On board the tanker, a flexible riser will be stored. This riser will be deployed through a moon-pool of the tanker. At the lower end of the riser, a Lower Riser Package (LRP) will be connected. This package includes an underwater navigation system, a thruster package, a power distribution system and a docking assembly.

The fiexible riser end and the LRP will be lowered through the moon-pool towards the seaffoor terminal to dock and latch onto a re-entry guide structure of the seaffoor terminal. The final approach to the seafloor terminal is made possible by the hydroacoustic navigation system and the thruster package which controls the motion of the LRP and the lower riser end position. A schematic arrangement of the SUBLOAD system is shown in Fig. 1.

This paper is concerned with the design of some of the crucial LRP parameters such as the weight, buoyancy, metacentre and thruster configuration of the LRP.

Received 30 April 1985.

† Kongsberg Albatross a.s., N-3600 Kongsberg, Norway. 


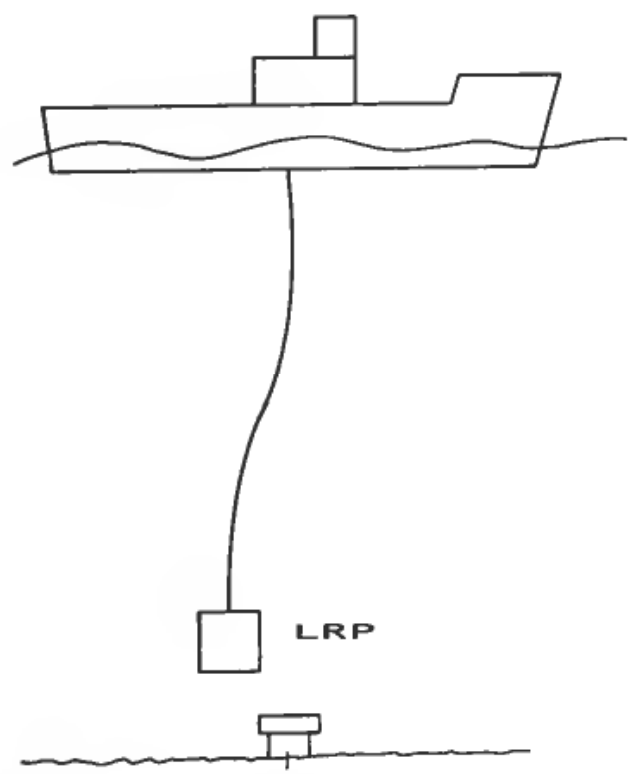

Figure 1. Schematic drawing of the SUBLOAD system.

This is done in order to minimize the thrust force requirement for docking control purposes. The parameters are found by means of a mathematical model of the vessel, the riser, the LRP and the environmental forces.

Secondly, a docking control system is designed based on a simplified model of the LRP dynamics. The control system uses feedback from LRP-states, as well as feedforward from estimated force components acting directly on the LRP. The system is adaptive in the sense that the dominating wave force frequency is continuously estimated and used by the control algorithm.

Finally, the control system is tested against a realistic simulator and the control performance is shown to be superior compared to a more direct PID based control system.

\section{Mathematical simulation model}

The mathematical model used for simulation consists of a riser model, an LRP model, a water wave model, and a model of the tanker.

\subsection{The riser model}

The riser model is based on the linear partial differential equation for a beam column with lateral loads in a vertical plane (Otteren 1982). The discussion is restricted to a two-dimensional model. This can be done because very little coupling exists between motions in two perpendicular planes. The riser model in the $x-z$ plane becomes:

$$
\begin{aligned}
& M_{R} \frac{\partial p}{\partial t}+\frac{\partial^{2}}{\partial z^{2}}\left(E I \frac{\partial^{2} x}{\partial z^{2}}\right)-\frac{\partial}{\partial z}\left(T \frac{\partial x}{\partial z}\right)=F \\
& \frac{\partial x}{\partial t}=p
\end{aligned}
$$




$$
\begin{aligned}
& F=\frac{1}{2} \rho_{W} C_{D}|u-p|(u-p)+\frac{1}{4} \rho_{W} \pi D^{2} C_{M} \frac{\partial u}{\partial t} \\
& T=g\left(W_{\text {LRP }}+W_{R} z\right)
\end{aligned}
$$

where

$x \quad$ horizontal riser deflection

$z \quad$ vertical co-ordinate (origin at the lower riser end)

$p \quad$ horizontal riser velocity

$M_{R} \quad$ mass + added mass per unit length of riser

EI bending stiffness

$T$ riser tension

$F \quad$ hydrodynamic force acting on the riser

$\rho_{W} \quad$ water density

$D \quad$ outer riser diameter

$C_{D} \quad$ drag coefficient of riser

$u \quad$ water velocity due to current and waves

$C_{M} \quad$ inertia coefficient for a fixed cylinder in an accelerating flow

$g$ gravitational constant

$W_{\text {LRP }}$ weight of the LRP in water

$W_{R} \quad$ weight per unit length of riser in water

The vertical motion of the riser is disregarded. In the SUBLOAD system this motion is counteracted by a motion compensation system on board the shuttle tanker.

The boundary conditions for the riser equation are:

$$
\begin{array}{cc}
\text { At the surface }(z=H): & p(H)=u_{T} \\
\text { At the riser end }(z=0): & p(0)=u_{L}
\end{array}
$$

where $u_{T}$ is the tanker velocity and $u_{L}$ is the LRP velocity at the joining point between the riser and the LRP.

\subsection{The LRP model}

The lower riser package is modeled as a rigid body connected to the riser via a perfectly flexible joint. A drawing of the geometry is shown in Fig. 2. The equations of motion can be written

$$
\begin{aligned}
\dot{u}_{L}= & \frac{1}{M^{*}}\left[W_{\mathrm{LRP}} g \sin \left(\frac{d x}{d z}\right)+d_{x}\left|u(0)-u_{L}\right|\left(u(0)-u_{L}\right)+F_{T}\right] \\
\dot{x}_{L}= & u_{L} \\
\dot{r}= & \frac{1}{I^{*}}\left[-W_{\mathrm{LRP}} L g \sin \left(\frac{d x}{d z}+\phi\right)-R_{M} \Delta V g \sin \phi\right. \\
& \left.-d_{x}\left|u(0)-u_{L}\right|\left(u(0)-u_{L}\right) R_{D}-d_{\phi}|r| r+M_{T}\right] \\
\dot{\phi}= & r
\end{aligned}
$$




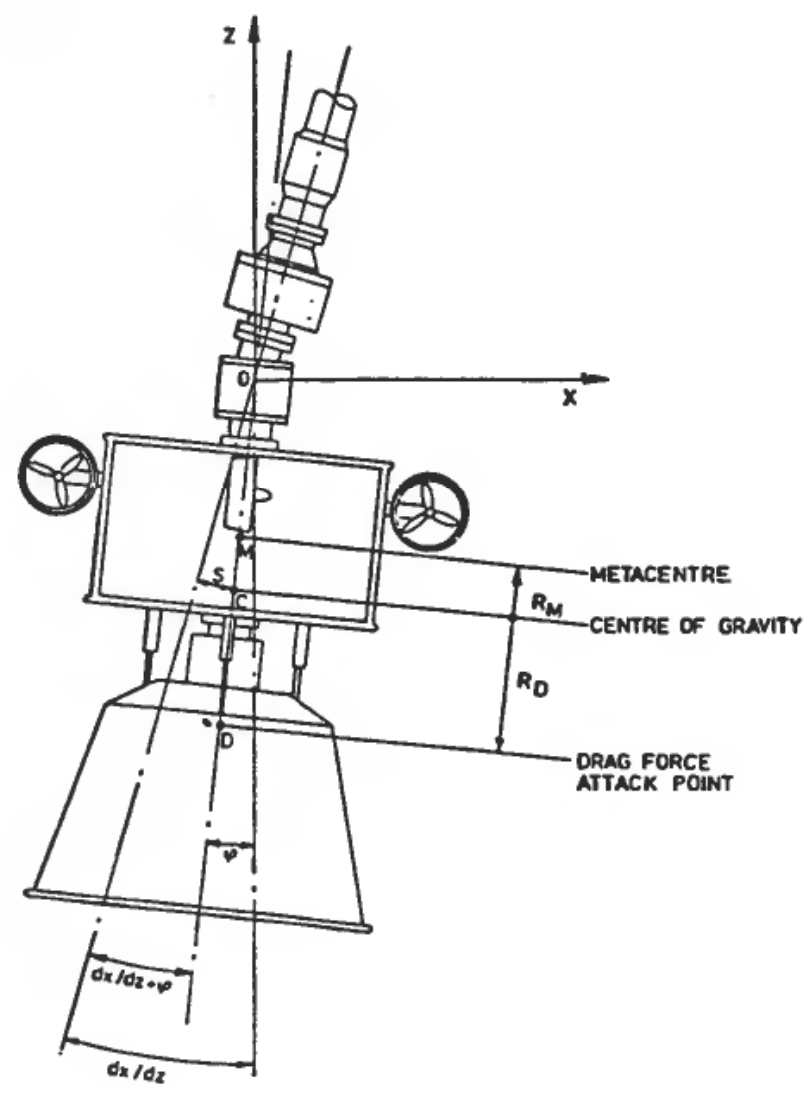

Figure 2. The geometry of the Lower Riser Package (LRP).

where

$x_{L} \quad$ horizontal position of the LRP centre of gravity

$M^{*} \quad$ mass + added mass of LRP

$d_{x^{\prime}} d_{\phi}$ drag coefficient of the LRP

$F_{T} \quad$ lateral thrust force acting on the LRP

$L \quad$ distance between flex-joint and centre of gravity

$\phi, r \quad$ roll angle and roll rate of the LRP

$I^{*} \quad$ moment of inertial + added moment of inertial of the LRP

$R_{M} \quad$ distance between centre of gravity and metacentre

$\Delta V \quad$ volume of LRP

$M_{T} \quad$ thrust moment acting on LRP

$R_{D} \quad$ distance between drag force attack point and centre of gravity

\subsection{The current, wave and tanker model}

The current is assumed to be given by a constant linear profile. The wave model is assumed to be the same as described in Ottern (1982). The horizontal water velocity is given by

$$
\begin{gathered}
u(z, t)=\sum_{m-1}^{N} A_{m \omega_{m}} \exp \left(-\frac{\omega_{m}^{2}}{g}(H-z)\right) \cos \left(\omega_{m} t+\phi_{m}\right) \\
A_{m}=\sqrt{ }\left[2 S\left(\omega_{m}\right) \Delta \omega\right]
\end{gathered}
$$


where deep water waves are assumed (Newman 1978) and where $u(z, t)$ is the horizontal water velocity, $\omega_{m}$ are discretization frequencies of a discretized water wave spectrum, $\Delta \omega$ is the frequency discretization interval, $H$ is the water depth, $\phi_{m}$ are random constant phases and $S(\omega)$ is the wave spectrum.

The vessel model is given by a simple non-linear model and the response of the vessel is found by application of the surface water wave to this vessel model.

\subsection{Simulation and verification of the total model}

A riser simulation model is produced by application of a semi-implicit finite difference method to the riser equations. A semi-implicit method is also applied to the LRP-equations. The method is illustrated by the LRP roll equations.

$$
\begin{aligned}
r^{k+1} & =r^{k}+M^{k} \Delta t \\
\phi^{k+1} & =\phi^{k}+\Delta t r^{k+1}
\end{aligned}
$$

where $k$ represents the discrete time index, $\Delta t$ is the discretization time interval, and $M^{k}$ is an approximation to the right hand side of eqn. (7) evaluated at time $k$.

The $z$-coordinate is discretized in $N$ points. $N=30$ is found to be appropriate for the given riser when $H=135$ metres. It turns out that $\Delta t$ should be chosen less than $0 \cdot 1$ seconds.

A scale model of the tanker/riser/LRP system in the linear scale 1:12.5 has been built and tested at the Norwegian Hydrodynamic Laboratories at the Norwegian Institute of Technology.

\section{Optimization of LRP design parameters}

Initially the riser/LRP system was designed without specific regard to the dynamic positioning requirements relative to the sea floor terminal of the LRP system. This section examines how some of the LRP parameters can be changed in order to minimize the thrust installation requirements. We will assume that the riser design parameters are fixed and cannot be changed.

\subsection{Important LRP-parameters}

In order to find the dynamically important parameters, we will examine the different terms of the LRP equations. We shall assume the following nominal parameters of the $\mathrm{LRP} /$ riser system:

$$
\begin{array}{rlrl}
M_{R} & =500 \mathrm{~kg} / \mathrm{m} & I^{*} & =54,300 \mathrm{~kg} / \mathrm{m}^{3} \\
H & =135 \mathrm{~m} & M^{*} & =37,300 \mathrm{~kg} \\
E I & =\text { negligibly small } & d_{x} & =8400 \\
\rho_{W} C_{d} & =800 \mathrm{~kg} / \mathrm{m}^{3} & d_{\phi} & =56,700 \\
D & =0.5 \mathrm{~m} & R_{M} & =0.13 \mathrm{~m} \\
C_{M} & =2.0 & R_{D} & =-0.5 \\
g & =9.81 \mathrm{~m} / \mathrm{s}^{2} & \Delta V & =6.0 \mathrm{~m}^{3} \\
W_{\mathrm{LRP}} & =19,000 \mathrm{~kg} & L & =2.0 \mathrm{~m} \\
W_{r} & =175 \mathrm{~kg} / \mathrm{m} & W_{\text {AIR }} & =25,000 \mathrm{~kg}
\end{array}
$$

where $W_{\text {AIR }}=W_{\text {LRP }}+\Delta V \cdot \rho_{W}$ is the weight in air of the LRP. 
By analysing eqns. (5) and (7), and by comparison of the relative size of the different terms, we can conclude that the potentially important parameters with respect to dynamic response are $W_{\mathrm{AIR}}, W_{\mathrm{LRP}}, R_{M}, L, d_{x}, R_{D}, I^{*}$ and $M^{*}$. These parameters are highly interconnected in the sense that they cannot be changed independently, and a parameter combination cannot be chosen freely.

To avoid this problem, we assume for simplicity that the volume of the LRP is defined by a cylindrical shape. Of course, this is not really so, but this is assumed as a simple model which makes possible the following design computations. We also assume that the LRP has an equivalent drag area used for computation of $d_{x}$ and $d_{\phi}$. This drag area is assumed different from the projected cylindrical shape. This is done because the drag area may be independently increased (by fins, etc.), without increasing the volume contributing to buoyancy. This conceptual model yields:

$$
\begin{aligned}
W_{\mathrm{LRP}} & =W_{\mathrm{AIR}}-R^{2} V \rho_{W} \\
M^{*} & =W_{\mathrm{AIR}}+k_{A} \pi R^{2} V \rho_{W} \\
I^{*} & =M^{*}\left(\frac{R^{2}}{4}+\frac{V^{2}}{12}+R_{M}^{2}\right) \\
d_{x} & =B_{D} V_{D} C_{D} \cdot \rho_{W} \\
d_{g} & =\frac{1}{32} d_{x} V_{D}^{3}
\end{aligned}
$$

where $\pi R^{2} V$ is the assumed LRP volume, $R$ is the radius of the assumed LRP cylinder, $V$ the height of this cylinder, $k_{A}$ is an added mass parameter, $B_{D}$ is the horizontal dimension of the drag area and $V_{D}$ is the vertical dimension of the drag area.

Due to design restrictions we assume that $L$ cannot be changed. The design restrictions on the remaining parameters are chosen as

$W_{\text {AIR }}: 25,000 \mathrm{~kg} \leqslant W_{\text {AIR }}<40,000 \mathrm{~kg}$

$R: \quad$ This effective radius may be increased by enclosing the LRP in a cylindrical shell (or another shape). This volume may be partly or totally filled with air which again increases the buoyancy. We assume that $0.7 \mathrm{~m} \leqslant R \leqslant 1.5 \mathrm{~m}$.

$V_{D}: \quad 5 \mathrm{~m} \leqslant V_{D} \leqslant 6 \mathrm{~m}$

$B_{D}: \quad 3 \mathrm{~m} \leqslant B_{D} \leqslant 6 \mathrm{~m}$

$R_{M}$ : The centre of buoyancy forces may be changed by mass arrangements.

$$
-1 \mathrm{~m} \leqslant R_{M} \leqslant 1 \mathrm{~m}
$$

$R_{D}: \quad-1.5 \mathrm{~m} \leqslant R_{D} \leqslant 1.5 \mathrm{~m}$

\subsection{Nominal case simulation}

In order to determine the effect of parameter variations, a nominal case simulation is taken as a basis for comparison. The environmental condition is given by a water wave time series having a significant wave height of $H_{1 / 3}=8$ metres and vessel excursions of $0.5-1.0$ metres in the surge direction. The vessel motion and the water wave amplitudes at different depths are shown in Fig. 3. The resulting linear and angular motions of the LRP are shown in Fig. 4.

We observe that the water waves induce a quite large motion amplitude of the LRP, and that the low frequency vessel motion is more or less directly transmitted to the LRP. 

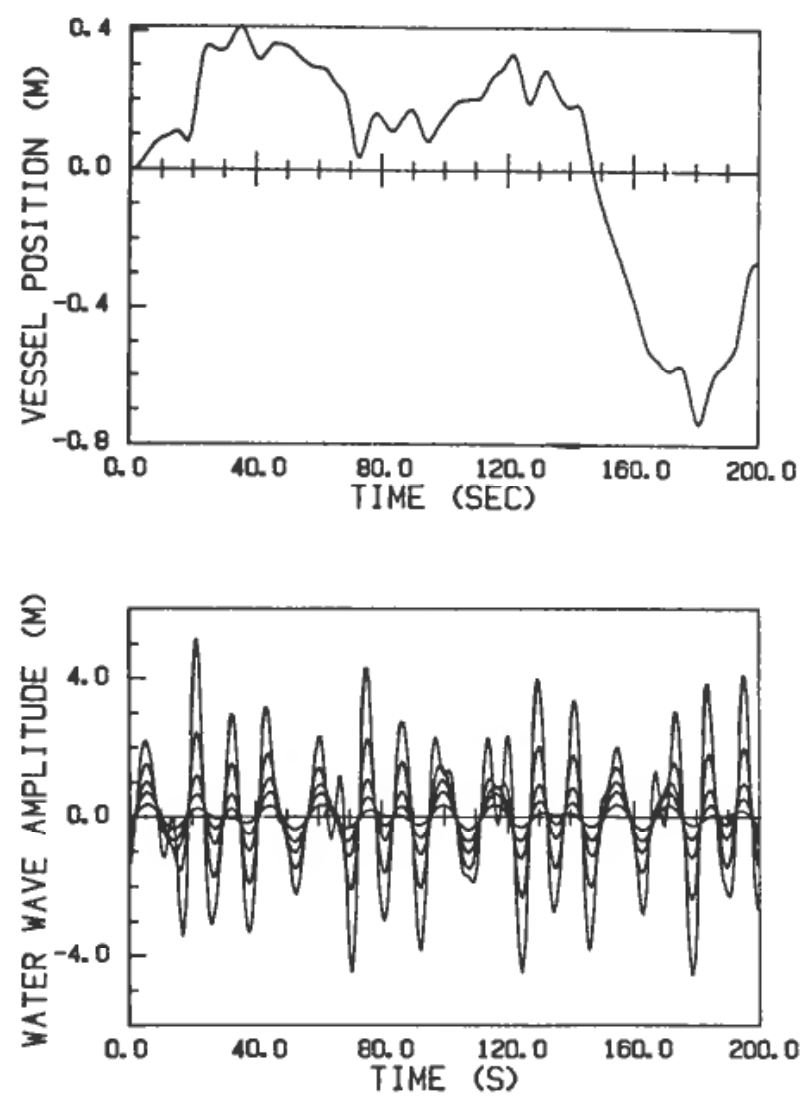

Figure 3. Vessel motion and water wave amplitudes at the surface and at the depths $23 \mathrm{~m}$, $46 \mathrm{~m}, 70 \mathrm{~m}, 93 \mathrm{~m}$, and $116 \mathrm{~m}$.

\subsection{Parameter sensitivity}

To determine which are the important parameters, we have performed a number of simulations using different values of $W_{\mathrm{AIR}}, R, V_{D}, B_{D}, R_{M}$ and $R_{D}$.

The simulations show the following facts:

An increase in $W_{\text {AIR }}$ mainly influences the riser horizontal motion amplitude by increasing the riser tension. Also the LRP roll angle and the LRP position are slightly changed. This is shown in Fig. 5.

The parameters $R_{D}, B_{D}$ and $V_{D}$ turn out to influence the LRP dynamics to a very small degree.

The parameter $R$ is a very important parameter. A change in $R$ induces a change in $W_{\mathrm{ARP}}$ and in $M^{*}$ and $I^{*}$. An increase in $R$ increases the buoyancy volume and $W_{\text {LRP }}$ will decrease. This will in turn decrease the riser tension and hence both the riser dynamics and the forces acting on the LRP from the riser. The LRP position and roll angle amplitudes together with the riser end angle amplitude are shown in Fig. 6. As seen, an increase in $R$ yields a decrease in the average LRP-position and a strong increase in the LRP and riser angle.

The parameter $R_{M}$ influences mainly the LRP roll angle dynamics. An increased $R_{M}$ decreases the amplitude of the roll motion.

This analysis reduces the important parameters to $W_{\mathrm{AIR}}, R$ and $R_{M}$. 

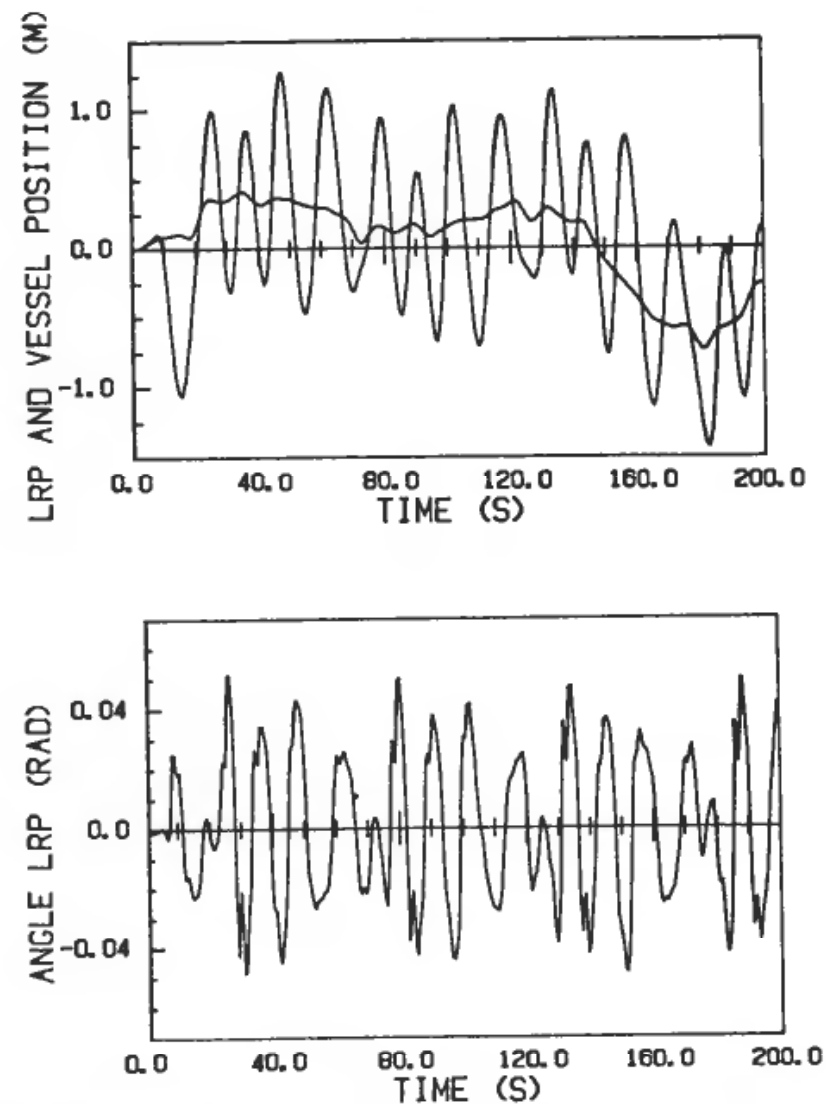

Figure 4. Linear and angular LRP motion shown for nominal parameter case. The vessel motion is also shown.

\subsection{Choice of parameters}

$W_{\text {AIR }}$ is limited by construction and handling constraints to be in the range $W_{\text {AIR }}$ $[25,000 \mathrm{~kg}, 40,000 \mathrm{~kg}]$.

The value of $R$ cannot be decreased below $0.7 \mathrm{~m}$, which is the nominal value. It can, however, be increased by designing the LRP as a partly air-filled space. This is indicated in Fig. 7 . The radius $R$ will in fact have an upper limit given by $W_{\text {LRP }}$ rather than geometry.

In order to avoid dangerous vertical motions of the LRP because of a nonperfect heave compensation system, $W_{\text {LRP }}$ should be greater than $10-15 \%$ of the
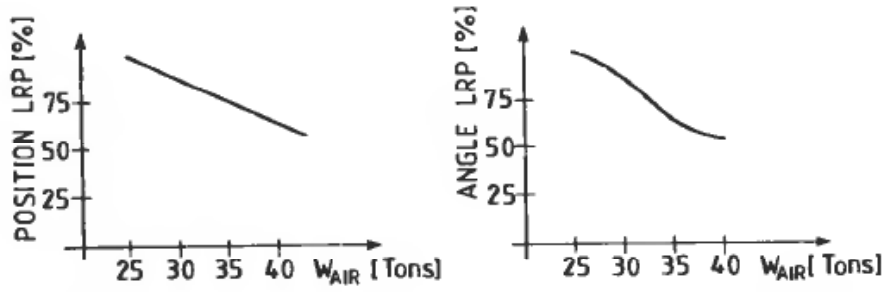

Figure 5. Average position and angle amplitude of the LRP as a function of $W_{\text {AIR }}$ (in $\%$ of nominal value). 

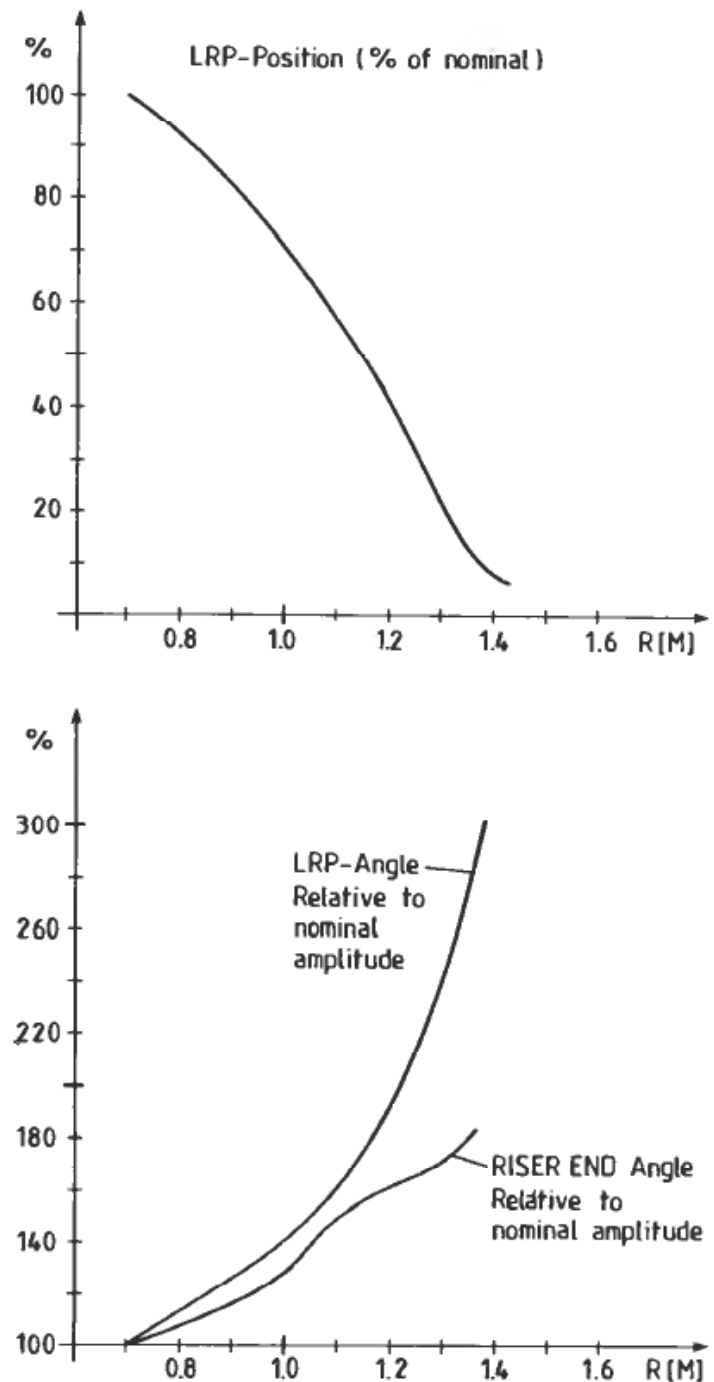

Figure 6. Relative LRP-position amplitude, LRP-angle amplitude and riser end angle amplitude as a function of $R$ (buoyancy).

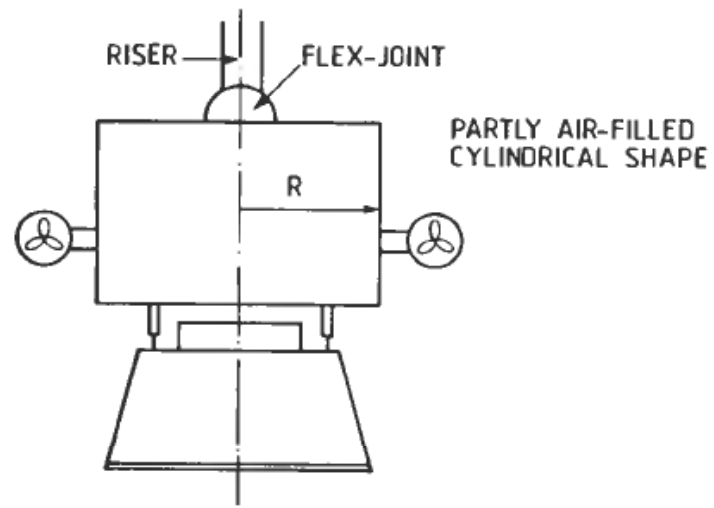

Figure 7. LRP designed within a partly air filled cylinder. 


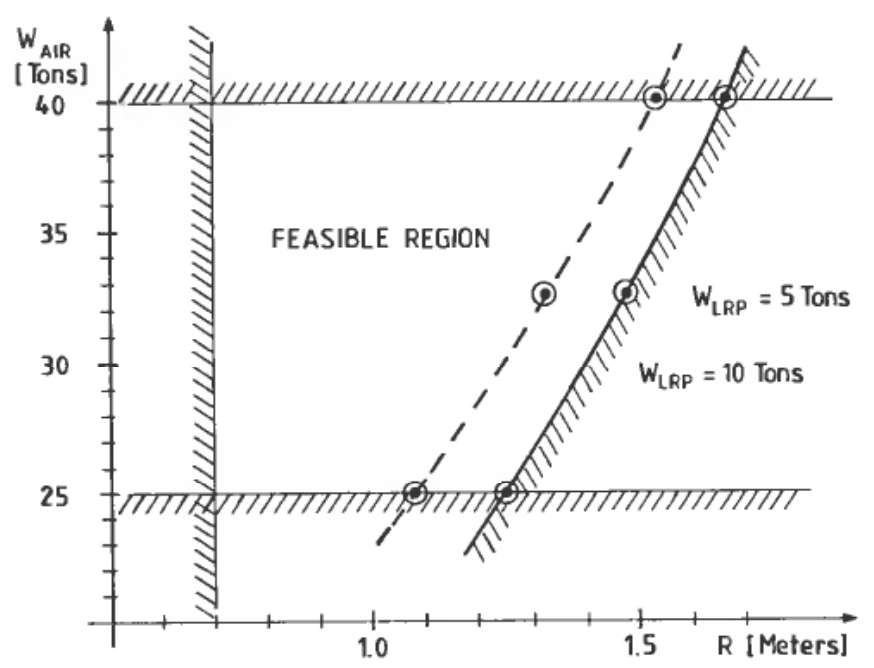

Figure 8. Feasible region for values of $W_{\mathrm{AIR}}$ and $R$.

surface riser tension. This means that $W_{\mathrm{LRP}} \geqslant 5000 \mathrm{~kg}$. Equation (13) yields

$$
W_{\text {AIR }}-\pi R^{2} V \rho_{W} \geqslant 5000
$$

With $V$ and $\rho_{W}$ given, the feasible region for combinations of $W_{\text {AIR }}$ and $R$ are shown in Fig. 8.

The 'optimal' choice of parameters from a control point of view is the set of parameters which minimizes the motion amplitudes. If we look at the LRP position only, it is quite clear from Figs. 5 and 6 that $W_{\text {AIR }}$ and $R$ should both be maximized within the given restrictions. However, when $R$ is increased, the LRP roll angle tends to increase strongly, as indicated in Fig. 6. Now, we have previously found that increasing the parameter $R_{M}$ reduces the roll angle amplitude. It turns out that by increasing $R_{M}$, the roll angle will not increase at all when $R$ and $W_{\text {AIR }}$ are maximized. The best choice of parameters from a dynamic point of view is therefore found by

Maximizing $R$ ( $W_{\mathrm{AlR}}$ given)

Maximizing $W_{\mathrm{AIR}}$

Maximizing $R_{M}$

This gives the values $R=1.67 \mathrm{~m}, W_{\mathrm{AIR}}=40,000 \mathrm{~kg}$, and $R_{M}=1.0 \mathrm{~m}$. From simulations using parameters along the right parabolic boundary in Fig. 8, it is seen that the choice of $W_{\text {AIR }}$ is rather unimportant. Because $W_{\text {AIR }}$ should be small from a handling point of view, $W_{\text {AIR }}$ is chosen equal to $25,000 \mathrm{~kg}$. This corresponds to $R=1.25$ at the parabolic boundary in Fig. 8. The final parameter choice is therefore given by

$$
R=1.25 \mathrm{~m}, W_{\mathrm{AIR}}=25,000 \mathrm{~kg}, R_{M}=1.0 \mathrm{~m} .
$$

The LRP position and roll angle for the new design parameters are shown for a 200 second simulation run in Fig. 9. By comparing with Fig. 4, we observe that the LRP horizontal position amplitude due to waves are reduced with as much as $\sim 70 \%$. The LRP angle has approximately the same average amplitude as in the nominal case. 

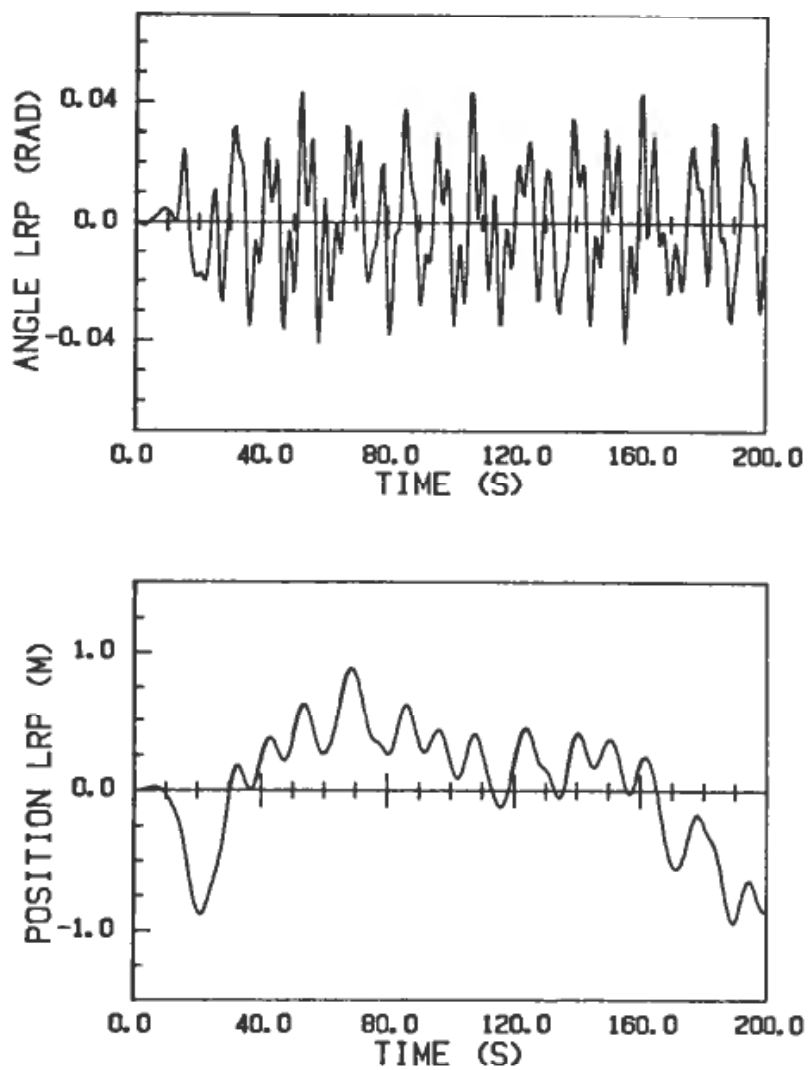

Figure 9. LRP roll angle and position for the optimal design. by

The docking mechanism of the LRP will have a linear horizontal motion given

$$
x_{D}=x_{L}+\phi L
$$

where the distance from the centre of gravity to the docking mechanism is assumed to be equal to $L$. If superscript $m$ indicates maximum values, we have

$$
x_{D}^{m}=x_{L}^{m}+\phi^{m} L
$$

With typical values inserted for the wave induced component, we obtain

$$
x_{D}^{m}=0.35+0.04 \cdot 2=0.43 \mathrm{~m}
$$

In the next section we shall see how a thruster control system can be designed in order to further reduce $x_{D}^{m}$.

\section{Control system}

In order to control the linear motion of the LRP, we will first introduce a PIDcontroller taking feedback from the LRP position to the applied thrust force. The controller has the form

$$
u=-\left[K_{p}\left(x-x_{R}\right)+K_{D} \frac{d x}{d t}+K_{I} \int\left(x-x_{R}\right) d t\right]
$$


where $u$ is the thrust force in Newton, $x$ is the position of the LRP centre of gravity, $x_{R}$ is the position set point, $K_{p}$ is the proportional gain, $K_{D}$ is the derivative gain and $K_{I}$ the integral gain. $K_{p}, K_{D}$ and $K_{I}$ are chosen as follows:

$K_{p}=3000 \mathrm{~N} / \mathrm{m}$. This yields a force of 3000 Newton when $x-x_{R}=1 \mathrm{~m}$ and $K_{I}=d x / d t=0$.

$K_{D}=\sqrt{ }\left(2 M^{*} K_{p}\right)$. This yields critical damping of the control loop if the LRP is regarded as a mass-system without external forces from waves, current and riser.

$K_{I}=100 \mathrm{~N} / \mathrm{ms}$. The given choice of $K_{p}$ and $K_{D}$ yields a response time constant of $\approx 10$ seconds.

The given integral gain gives an integral time of $\approx 30$ seconds. Hence the integral part should not interfere with the PD-action of the controller.

This PID-controller is tested against the simulator of the vessel/riser/LRP system. The optimal parameter set $R=1.25 \mathrm{~m}, R_{M}=1 \mathrm{~m}$, and $W_{\mathrm{AIR}}=25$ tons are used. A simulation is performed using the previously defined wave excitations and a linearly varying current profile which goes from $0.4 \mathrm{~m} / \mathrm{s}$ at the bottom to $1 \mathrm{~m} / \mathrm{s}$ at the surface. The thrust force is assumed to apply at the centre of gravity of the LRP. Simulation results are shown in Fig. 10. The LRP angle amplitude is seen to be $\approx 0.015$ radians and the peak LRP position amplitude $\approx 0.25 \mathrm{~m}$. Hence the total motion amplitude of the docking mechanism becomes

$$
x_{D}^{m}=(0 \cdot 25+0 \cdot 015 \cdot 2) \mathrm{m}=0.28 \mathrm{~m}
$$

The DC-component of the applied thrust force can in a practical system be removed by changing the set point of the dynamically positioned surface vessel. Hence the thrust requirements are indicated by the peak AC-value of the thrust demand. This value is seen to be $\approx 1000$ Newton. The simulation represents an average weather condition, and the thruster should be designed for a maximum thrust force of approximately 3000 Newton.

The proposed control solution does not explicitly use the knowledge that the main disturbances acting on the LRP are oscillatory in nature. An alternative control system based on a simple mathematical model of the LRP and the disturbances is therefore proposed. The assumed model has the form

$$
\begin{aligned}
& \dot{x}_{1}=x_{2} \\
& \dot{x}_{2}=\frac{1}{M^{*}}\left(x_{3}+x_{5}+u\right)+\eta_{1} \\
& \dot{x}_{3}=x_{4}+\eta_{2} \\
& \dot{x}_{4}=-\omega^{2} x_{3} \\
& \dot{x}_{5}=\eta_{3}
\end{aligned}
$$

where

$x_{1} \quad$ LRP position [m]

$x_{2} \quad$ LRP velocity $[\mathrm{m} / \mathrm{s}]$

$x_{3}$ oscillatory disturbance force [Newton]

$x_{4}$ rate of change of oscillatory disturbance force $[\mathrm{N} / \mathrm{sec}]$

$x_{5}$ slowly varying disturbance force [Newton]

$\eta_{1}, \eta_{2}, \eta_{3}$ white process noise, $\boldsymbol{\eta}=\left\{\eta_{\mathrm{i}}\right\}$ 

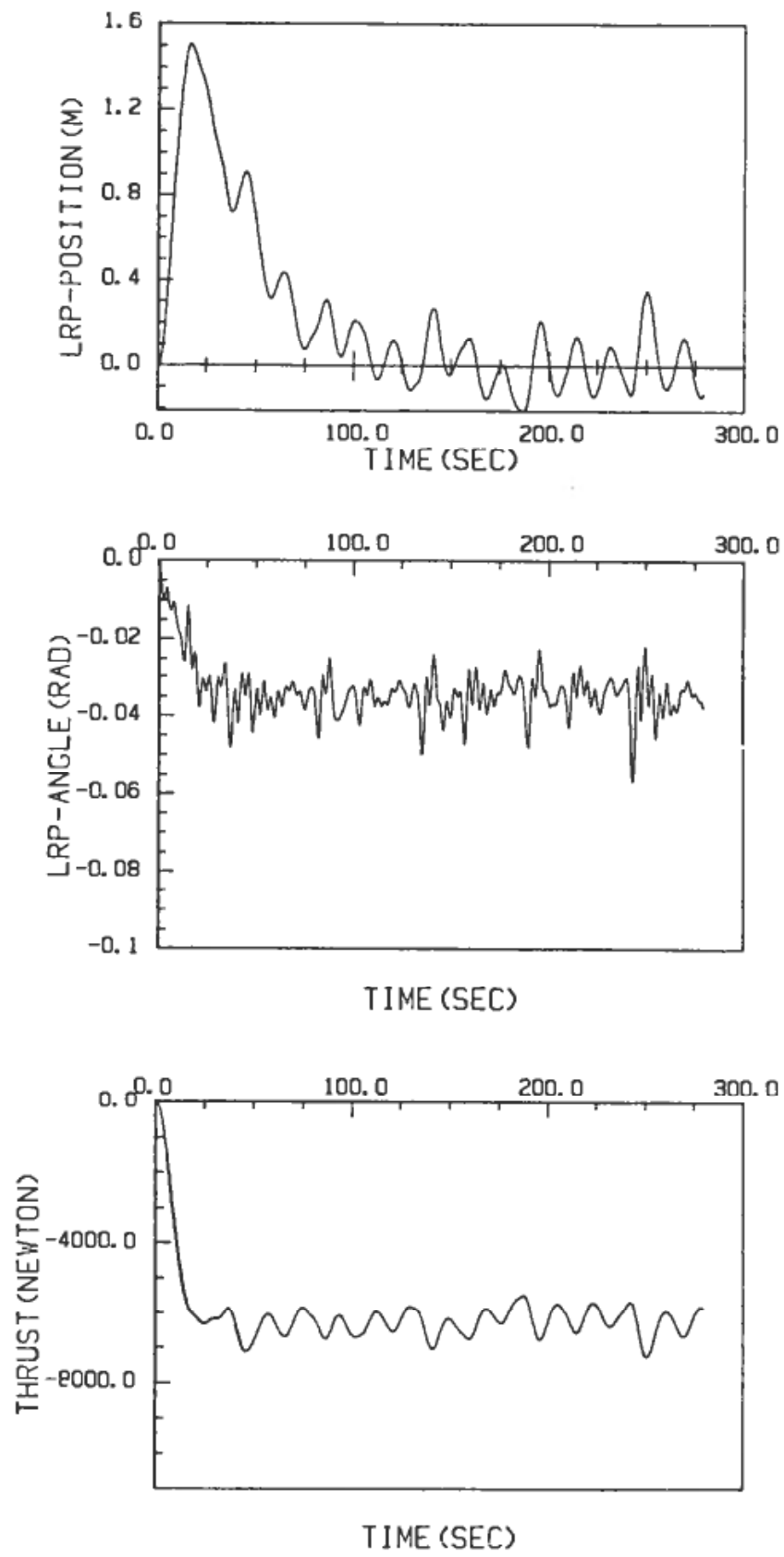

Figure 10. LRP-behaviour and applied thrust force in the PID controller case.

$M^{*}$ mass + added mass of LRP [kg]

$\omega$ dominating wave frequency $[\mathrm{rad} / \mathrm{sec}]$

$u$ thrust force [Newton]

Note that eqns. (22) and (23) make up an oscillatory system which is intended to simulate the oscillatory external forces. Now $x_{1}$ is assumed to be measured as $y=$ $x_{1}+w$, where $w$ is white measurement noise. This model is used in a steady state 
Kalman filter (Jazwinski 1970) for estimation of the states. The process and measurement noise covariance matrices are chosen as $\operatorname{cov}(w)=2 \cdot 10^{-4}$, $\operatorname{cov}(\eta)=\left\{10^{-5}, 10^{4}, 10^{3}\right\}$. In addition to the Kalman filter, a recursive parameter estimator is introduced in order to estimate the dominating wave frequency of the oscillatory disturbances. A prediction error algorithm similar to that described by Saelid et al. (1983) is applied. The algorithm on discrete form is

$$
\begin{aligned}
\hat{\omega}_{k+1} & =\hat{\omega}_{k}-P_{k} \psi_{k} \varepsilon_{k} \\
P_{k+1} & =\frac{1}{\lambda}\left[P_{k}-p_{k}^{2} \psi_{k}^{2} /\left(1+\psi_{k}^{2} P_{k}\right)\right]
\end{aligned}
$$

$\varepsilon_{k}$ is the Kalman filter innovation estimate of $\hat{\omega}_{k}, \lambda$ is a forgetting factor, and $\psi_{k}=$ $\partial \varepsilon_{k} / \hat{\omega}_{k}$ is a sensitivity function which is found by solving a set of sensitivity equations. See Saelid et al. (1983).
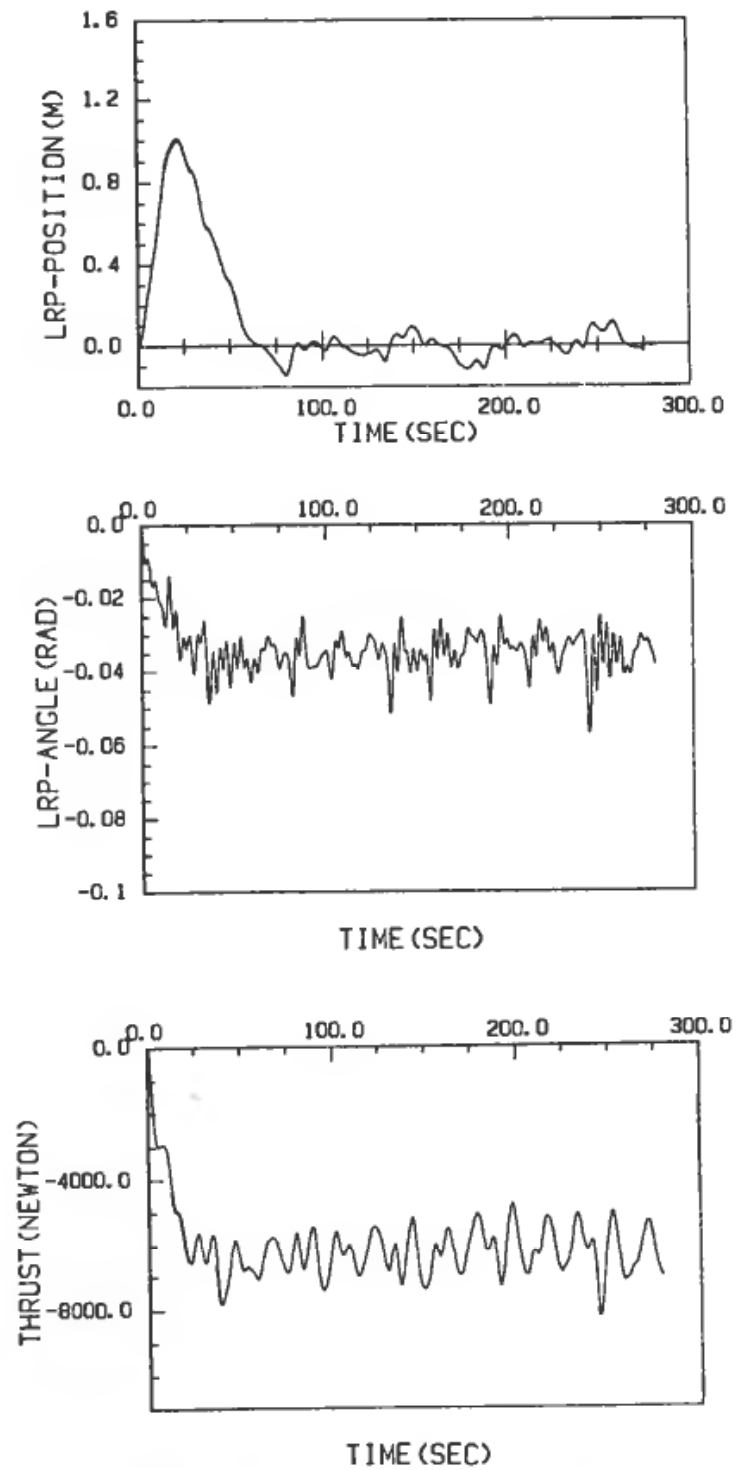

Figure 11. LRP-behaviour and applied thrust in the model based controller case. 
The controller now has the form

$$
u=-\left[K_{p}\left(\hat{x}_{1}-x_{1 R}\right)+K_{D}\left(\hat{x}_{2}-x_{2 R}\right)+\hat{x}_{3}+\hat{x}_{5}\right]
$$

where $x_{1 R}$ is the position set point, $x_{2 R}$ is the velocity reference, and $\hat{x}_{i}$ is the Kalman filter estimate of $x_{i}$. Here, the $\hat{x}_{3}$ term compensates for the oscillatory disturbance force while the $\hat{x}_{5}$ term provides the integral action of the system. The gains $K_{P}$ and $K_{D}$ are chosen as in the PID controller. The performance of this control system is shown in Fig. 11.

We observe that the LRP angle amplitude is still $\simeq 0.015$ radians, but the position amplitude has decreased to $\approx 0.1 \mathrm{~m}$. Hence the total motion amplitude becomes

$$
x_{D}^{m}=(0 \cdot 1+0.015 \cdot 2) \mathrm{m}=0 \cdot 13 \mathrm{~m}
$$
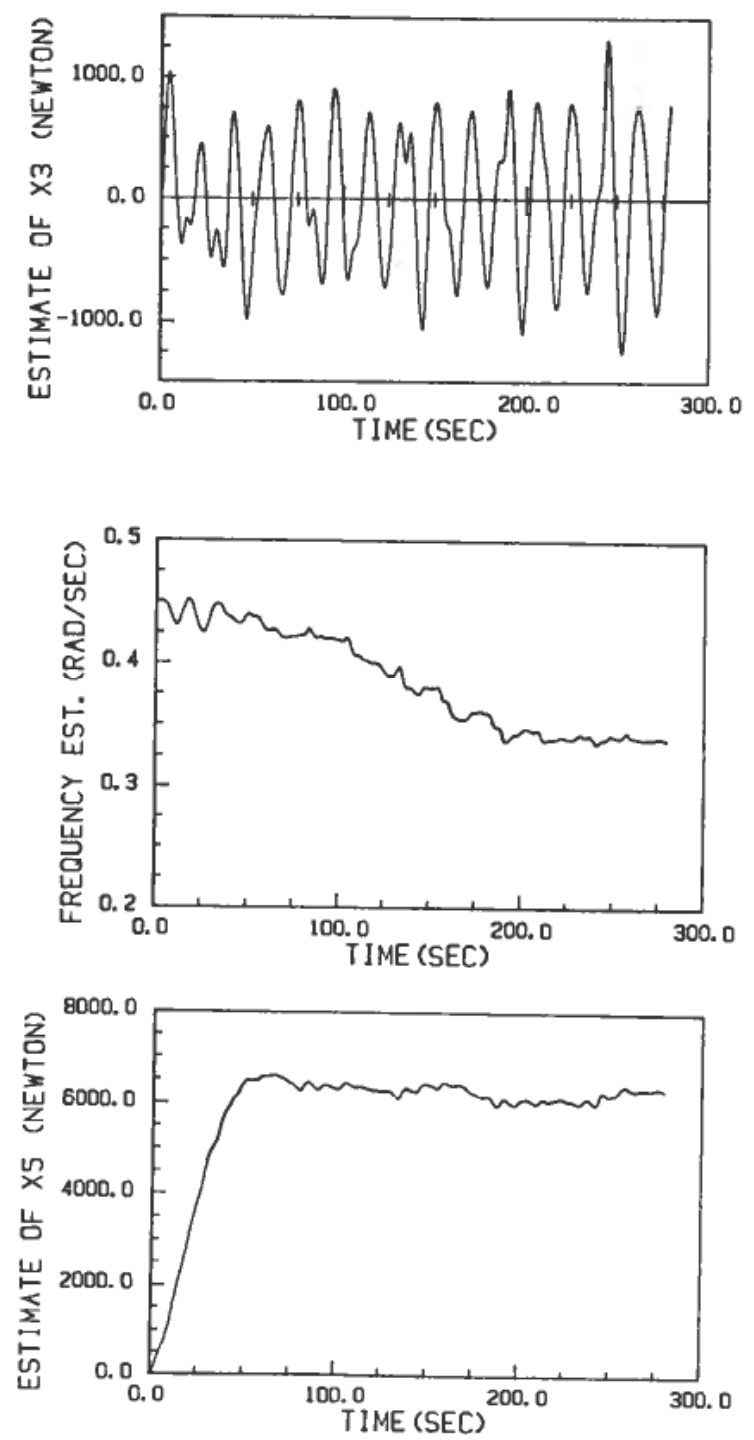

Figure 12. Estimates of the oscillatory force $\left(x_{3}\right)$, the slowly variable force $\left(x_{5}\right)$ and the oscillation frequency. 
The AC component of the thrust has now increased to a peak value of $\approx 1500$ Newton. As seen, this is a far better control system than the PID based system. This is due to the estimation of the external force by using a mathematical model of how this force is generated. The estimate of $x_{3}, x_{5}$, and $\omega$ are shown in Fig. 12.

\section{Conclusion}

We have in this paper presented an example of how mathematical modeling can be applied in order to optimize the design parameters of a system with respect to control behaviour. In the SUBLOAD system the installed thrust could be reduced by more than $50 \%$ compared to the initial design where the problems of control performance were not so explicitly addressed. In the design analysis, a rather detailed model of the system was applied. For actual control purposes, a control system based on a simplified system model has been proposed and shown by simulations to be far superior to an ordinary PID based control system.

\section{ACKNOWLEDGMENT}

The author wishes to thank Kongsberg Engineering $\mathrm{A} / \mathrm{S}$ for permission to publish this material.

\section{REFERENCES}

JAZWInSKI, A. H. (1970). Stochastic Processes and Filtering Theory. (New York, Academic Press).

Newman, J. N. (1978). Marine Hydrodynamics. (Cambridge, Mass., MIT Press).

OTTEREN, A. (1982). A mathematical model for dynamical analysis of a flexible marine riser connected to a floating vessel. Modeling, Identification and Control, 3, 187-209.

Saelid, S., JensSen, N. A., and BalChen, J. G. (1983). Design and analysis of a dynamic positioning system based on Kalman filtering and optimal control. I.E.E.E. Trans. auto. Control, 28, 331-339. 